PSI-PR-96-09

February 1996

\title{
Comparative Study of $Z^{\prime}$ Searches in $e^{+} e^{-}$and $e^{-} e^{-}$Scattering
}

\author{
Frank Cuypers \\ cuypers@pss058.psi.ch \\ Paul Scherrer Institute, CH-5232 Villigen PSI, Switzerland
}

\begin{abstract}
We consider indirect searches for additional neutral vector bosons in $e^{+} e^{-}$and $e^{-} e^{-}$collisions, and compare these two linear collider modes with similar analysis procedures and assumptions. Discovery limits and resolving power are discussed in a model-independent way.
\end{abstract}




\section{Introduction}

In contrast to storage rings, it is straightforward to replace a positron beam by an electron beam at a linear collider. There are several important characteristics which differentiate $e^{-} e^{-}$from $e^{+} e^{-}$collisions [1] and justify considering both options on the same footing when it comes to designing the linear colliders of the next generation, generically the Future Linear Collider (FLC):

- The $e^{-} e^{-}$environment is much cleaner because there is much less standard model activity. In particular, since QCD enters the game only at much higher orders and is associated with missing energy, the systematic errors due to the possible misidentification of electrons and pions are negligible.

- Current electron guns can already produce beams with polarizations exceeding $75 \%$. There is no doubt that even further improvements are due. It is not clear, however, whether polarized positron beams may ever be obtained at all. The $e^{-} e^{-}$collisions therefore offer the possibility of polarizing both initial states and to perform three independent experiments.

- The $e^{-} e^{-}$initial state is not only doubly charged, but also carries a finite lepton number. This allows to explore areas of new physics which are, at best, difficult to access in conventional $e^{+} e^{-}$annihilations.

We investigate here how a $Z^{\prime}$ can be discovered and studied in $e^{ \pm} e^{-}$scattering. For the $e^{+} e^{-}$reaction we concentrate on muon pair production [2] which provides a very clean signal, whereas for the $e^{-} e^{-}$process we analyze corrections to Møller scattering [3].

The next section is devoted to a short description of some models predicting the existence of a $Z^{\prime}$. After this, we discuss the expected $e^{ \pm} e^{-}$cross sections. We then proceed to a comparison of the discovery potential of each reaction, both at moderate SLC and LEP2 energies, as well as at the design FLC energy of $500 \mathrm{GeV}$. In the event a $Z^{\prime}$ is discovered, we examine how precisely the three parameters involved (vector and axial couplings and mass) can be measured and compare these expectations with the predictions of some models. Finally, we shortly comment on the incidence of radiative corrections.

\section{$2 \quad Z^{\prime}$ Models}

There are many extensions of the standard model which predict the existence of extra neutral vector bosons $Z^{\prime}$, and most searches are performed within the framework of a particular model [4]. It was advocated, though, that it is important to perform a model-independent analysis [2], in order not to miss out on some unexpected new kind of physics lurking beyond the standard 
model. We also adopt this point of view here.

Assuming lepton universality, the generic lagrangian describing the interaction of a heavy neutral vector boson $Z^{\prime}$ with the charged leptons can be written

$$
L=e \bar{\psi}_{\ell} \gamma^{\mu}\left(v_{Z^{\prime}}+a_{Z^{\prime}} \gamma_{5}\right) \psi_{\ell} Z_{\mu}^{\prime}
$$

where $v_{Z^{\prime}}$ and $a_{Z^{\prime}}$ are the vector and axial couplings normalized to the charge of the electron $e$. This interaction mediates both $e^{+} e^{-}$annihilation and $e^{-} e^{-}$ scattering. The results of LEP1 constrain any $Z^{0}-Z^{\prime}$ mixing to such an extent [5] that it can safely be ignored here.

In a model-independent analysis the two couplings in the lagrangian (11) are free. In the realm of specific models, however, their values are constrained or even fixed. We summarize now a few commonly used models, and will refer to these particular choices later, when it comes to gauge the capabilities of a linear collider operated in both $e^{+} e^{-}$and $e^{-} e^{-}$modes. Note that for all these models the $Z^{\prime}$ mass remains a free parameter. The trajectories or locations in the $\left(v_{Z^{\prime}}, a_{Z^{\prime}}\right)$ plane of these models are shown in Fig. 6

\subsection{Sequential Standard Model}

In this model it is assumed that there is a heavy $Z^{\prime}$ vector boson which couples to the fermions in the same way as the $Z^{0}$ :

$$
S S M:\left\{\begin{array}{l}
v_{Z^{\prime}}=v_{Z^{0}}=\frac{1-4 \sin ^{2} \theta_{w}}{4 \sin \theta_{w} \cos \theta_{w}} \\
a_{Z^{\prime}}=a_{Z^{0}}=\frac{-1}{4 \sin \theta_{w} \cos \theta_{w}} .
\end{array}\right.
$$

This model is neither realistic nor even gauge invariant, but some authors treat it as a useful benchmark.

\subsection{Un-unified Standard Model}

This model [6] assumes leptons and quarks transform according to different $S U(2)_{L}$ weak groups. The resulting $Z^{\prime}$ interacts then only with left handed fermions, and its couplings to the charged leptons are:

$$
U U:\left\{\begin{array}{ll}
v_{Z^{\prime}}=\frac{1}{2 \sin \theta_{w}} & \tan \phi \\
a_{Z^{\prime}}=-\frac{1}{2 \sin \theta_{w}} & \tan \phi
\end{array} \quad .22 \leq \sin \phi \leq .99,\right.
$$


where $\theta_{w}$ is the weak mixing angle.

\subsection{Left-Right Symmetric Models}

These models are based on the gauge group $S U(2)_{L} \otimes S U(2)_{R} \otimes U(1)$. This group can emerge as an intermediate symmetry from the breaking of a $S O(10)$ grand unified theory, in which case the $Z^{\prime}$ couplings to the charged leptons are [7]:

$$
L R:\left\{\begin{array} { l l } 
{ v _ { Z ^ { \prime } } = \frac { 1 } { 4 \operatorname { c o s } \theta _ { w } } } & { \frac { 2 - \beta ^ { 2 } } { \beta } } \\
{ a _ { Z ^ { \prime } } = - \frac { 1 } { 4 \operatorname { c o s } \theta _ { w } } } & { \beta }
\end{array} \left\{\begin{array}{l}
\beta=\sqrt{\frac{\kappa^{2}}{\tan ^{2} \theta_{w}}-1}>0 \\
\kappa=\frac{g_{R}}{g_{L}}>\tan \theta_{w}
\end{array}\right.\right.
$$

where $g_{L, R}$ are the couplings constants of the two $S U(2)$ groups. If these two couplings are equal, one talks of the manifestly left-right symmetric model (MLR).

In the so-called alternative left-right symmetric model the $S U(2)_{L} \otimes S U(2)_{R} \otimes$ $U(1)$ group originates as a subroup of $E_{6}$ [8]. The couplings to the charged leptons are then related to the above ones by the simple replacement $\beta \leftrightarrow$ $-1 / \beta$ and read

$$
A L R: \begin{cases}v_{Z^{\prime}}=\frac{1}{4 \cos \theta_{w}} & \frac{1-2 \beta^{2}}{\beta} \\ a_{Z^{\prime}}=\frac{1}{4 \cos \theta_{w}} & \frac{1}{\beta}\end{cases}
$$

Again, we define also the alternative manifestly left-right symmetric model (AMLR) by assuming $g_{L}=g_{R}$.

\subsection{Grand Unified Theories}

The linear combination of two independent $U(1)$ subgroups of a grand unified $E_{6}$ gauge group yield the following type of $Z^{\prime}$ couplings to the charged leptons [9]:

$$
G U T:\left\{\begin{array}{l}
v_{Z^{\prime}}=\frac{1}{\sqrt{6} \cos \theta_{w}} \quad \cos \beta \\
a_{Z^{\prime}}=-\frac{1}{2 \sqrt{6} \cos \theta_{w}}\left(\cos \beta+\sqrt{\left.\frac{5}{3} \sin \beta\right)} \quad-\pi \leq \beta \leq \pi .(6)\right.
\end{array}\right.
$$


There are some favoured values of the mixing angle $\beta$, which correspond to particular symmetry breaking chains. They are labeled $\psi, \chi$ and $\eta$ :

$$
\begin{array}{lll}
\psi: & \beta=\frac{\pi}{2} & \left(E_{6} \rightarrow S O(10) \otimes U(1)_{\psi}\right) \\
\chi: & \beta=0 & \left(S O(10) \rightarrow S U(5) \otimes U(1)_{\chi}\right) \\
\eta: & \tan \beta=-\sqrt{\frac{5}{3}} & \text { (superstring inspired) } .
\end{array}
$$

\section{Cross Sections}

The Born level Feynman diagrams for the $e^{+} e^{-} \rightarrow \mu^{+} \mu^{-}$and $e^{-} e^{-} \rightarrow e^{-} e^{-}$ reactions are shown in Figs 1. Neglecting the electron mass, the differential cross sections are

$$
\begin{aligned}
& \frac{d \sigma^{e^{+} e^{-}}}{d \cos \theta}=\frac{\pi \alpha^{2}}{4 s} \sum_{i, j} \frac{1}{\left(1-y_{i}\right)\left(1-y_{j}\right)} \\
& \left\{\frac{1-P}{2} \quad L_{i} L_{j}\left[L_{i} L_{j}(1-\cos \theta)^{2}+R_{i} R_{j}(1+\cos \theta)^{2}\right]\right. \\
& \left.+\frac{1+P}{2} \quad R_{i} R_{j}\left[R_{i} R_{j}(1-\cos \theta)^{2}+L_{i} L_{j}(1+\cos \theta)^{2}\right]\right\} \\
& \frac{d \sigma^{e^{-} e^{-}}}{d \cos \theta}=\frac{2 \pi \alpha^{2}}{s} \sum_{i, j} \frac{1}{\left(x_{i}^{2}-\cos ^{2} \theta\right)\left(x_{j}^{2}-\cos ^{2} \theta\right)} \\
& \left\{\frac{1-P_{1}-P_{2}+P_{1} P_{2}}{4} \quad L_{i}^{2} L_{j}^{2} \quad 8 x_{i} x_{j}\right. \\
& +\frac{1+P_{1}+P_{2}+P_{1} P_{2}}{4} \quad R_{i}^{2} R_{j}^{2} \quad 8 x_{i} x_{j} \\
& \left.+\frac{1-P_{1} P_{2}}{2} \quad R_{i} L_{i} R_{j} L_{j} \quad\left[x_{i} x_{j}+\left(1+x_{i} x_{j}+2 x_{i}+2 x_{j}\right) \cos ^{2} \theta+\cos ^{4} \theta\right]\right\} \\
& y_{i}=\frac{m_{i}^{2}}{s} \quad x_{i}=1+2 y_{i} \quad i, j=\gamma, Z^{0}, Z^{\prime} \\
& \left\{\begin{array} { l } 
{ R _ { i } = v _ { i } + a _ { i } } \\
{ L _ { i } = v _ { i } - a _ { i } }
\end{array} \quad \left\{\begin{array} { l } 
{ v _ { \gamma } = 1 } \\
{ a _ { \gamma } = 0 }
\end{array} \quad \left\{\begin{array}{l}
v_{Z^{0}}=\frac{1-4 \sin ^{2} \theta_{w}}{4 \sin \theta_{w} \cos \theta_{w}} \\
a_{Z^{0}}=\frac{-1}{4 \sin \theta_{w} \cos \theta_{w}}
\end{array}\right.\right.\right.
\end{aligned}
$$


The polarizations of the electron beams are given by $P$ and $\left(P_{1}, P_{2}\right)$, in the $e^{+} e^{-}(10)$ and the $e^{-} e^{-}$(11) reactions respectively. In our convention, a fully right(left) polarized beam is given by $P=1(-1)$.

We see that the $e^{+} e^{-}$cross section (10) is asymmetric, whereas the $e^{-} e^{-}$cross section (11) is symmetric in the scattering angle. The resulting total cross sections in the presence and absence of a $Z^{\prime}$ are shown for unpolarized beams in Fig. 2, where we imposed an angular cut of $10^{\circ}$ on the scattering angle. The total cross section of the $e^{-} e^{-}$reaction is very insensitive to the presence of a heavy $Z^{\prime}$, whereas the $e^{+} e^{-}$cross section is strongly influenced by the tail of the $Z^{\prime}$ resonance.

It is instructive to estimate these cross sections in the limit where

$$
m_{Z^{0}}^{2} \ll s \ll m_{Z^{\prime}}^{2} \quad \text { and } \quad \sin ^{2} \theta_{w}=\frac{1}{4} .
$$

With these approximations we have

$$
\left\{\begin{array} { l } 
{ y _ { \gamma } = y _ { Z ^ { 0 } } = 0 } \\
{ y _ { Z ^ { \prime } } = m _ { Z ^ { \prime } } ^ { 2 } / s } \\
{ x _ { \gamma } = x _ { Z ^ { 0 } } = 1 } \\
{ x _ { Z ^ { \prime } } = 2 m _ { Z ^ { \prime } } ^ { 2 } / s }
\end{array} \quad \left\{\begin{array} { l } 
{ v _ { e } = 1 } \\
{ a _ { e } = 0 } \\
{ v _ { Z ^ { 0 } } = 0 } \\
{ a _ { Z ^ { 0 } } = - 1 / \sqrt { 3 } }
\end{array} \quad \left\{\begin{array}{l}
R_{e}=1 \\
L_{e}=1 \\
R_{Z^{0}}=-1 / \sqrt{3} \\
L_{Z^{0}}=1 / \sqrt{3} .
\end{array}\right.\right.\right.
$$

It is then easy to compute the standard model contributions, who represent the bulk of the cross section:

$$
\begin{aligned}
& \frac{d \sigma^{e^{+} e^{-}}}{d \cos \theta}=\frac{\pi \alpha^{2}}{9 s}\left(5-6 \cos \theta+5 \cos ^{2} \theta\right) \\
& \frac{d \sigma^{e^{-} e^{-}}}{d \cos \theta}=\frac{8 \pi \alpha^{2}}{9 s} \frac{1}{\sin ^{4} \theta}\left[\frac{1+P_{1} P_{2}}{2} 32\right. \\
& \left.+\frac{1-P_{1} P_{2}}{2}\left(1+6 \cos ^{2} \theta+\cos ^{4} \theta\right)\right] \text {. }
\end{aligned}
$$

The $e^{-} e^{-}$cross section has thus always a strong polarization dependence. In contrast, the $e^{+} e^{-}$cross section should thus not depend very much on the electron beam polarization. 


\section{Discovery Limits}

For small $Z^{\prime}$ couplings or large $Z^{\prime}$ masses, the signal will mainly be confined in the interference terms. In the limits (14) we find

$$
\begin{aligned}
& \Delta \frac{d \sigma^{e^{+} e^{-}}}{d \cos \theta}=\frac{\pi \alpha^{2}}{3 m_{Z^{\prime}}^{2}} \quad\left\{\frac{1-P}{2} L_{Z^{\prime}}\left[2 L_{Z^{\prime}}(1-\cos \theta)^{2}+R_{Z^{\prime}}(1+\cos \theta)^{2}\right]\right. \\
& \left.+\frac{1+P}{2} R_{Z^{\prime}}\left[2 R_{Z^{\prime}}(1-\cos \theta)^{2}+L_{Z^{\prime}}(1+\cos \theta)^{2}\right]\right\} \\
& \Delta \frac{d \sigma^{e^{-} e^{-}}}{d \cos \theta}=\frac{4 \pi \alpha^{2}}{3 m_{Z^{\prime}}^{2}} \quad \frac{1}{\sin ^{2} \theta} \quad\left[16 R_{Z^{\prime}}^{2} \frac{1+P_{1}+P_{2}+P_{1} P_{2}}{4}\right. \\
& +16 L_{Z^{\prime}}^{2} \frac{1-P_{1}-P_{2}+P_{1} P_{2}}{4} \\
& \left.+R_{Z^{\prime}} L_{Z^{\prime}} \frac{1-P_{1} P_{2}}{2}\left(1+4 \cos ^{2} \theta\right)\right] \text {. }
\end{aligned}
$$

With fully polarized beams the $e_{R}^{-} e_{R}^{-}\left(P_{1}=P_{2}=1\right)$ and $e_{L}^{-} e_{L}^{-}\left(P_{1}=P_{2}=-1\right)$ reactions can probe independently $R_{Z^{\prime}}$ and $L_{Z^{\prime}}$. The third term in Eq. (19) is strongly suppressed and a measurement in the $e_{L}^{-} e_{R}^{-}\left(P_{1}=-P_{2}=1\right)$ mode will therefore not provide very much information. For this reason we shall ignore this combination of polarizations for the time being.

We can combine Eqs $(18,19)$ with Eqs $(16,18)$ to derive the Cramer-Rao bound on the smallest values of the $Z^{\prime}$ couplings one can observe in each experiment [10]:

$$
\chi_{\infty}^{2}=\mathcal{L} \int_{-1}^{+1} d \cos \theta \frac{\left(\Delta \frac{d \sigma}{d \cos \theta}\right)^{2}}{\frac{d \sigma}{d \cos \theta}}
$$

Assuming both experiments are performed with the electron beams first fully right polarized and then fully left polarized, we obtain the following quartic expressions for the highest possible resolution obtainable with an integrated luminosity $\mathcal{L}$

$$
\chi_{\infty}^{2}\left(e^{+} e^{-}\right) \simeq \mathcal{L} \quad \frac{\pi \alpha^{2} s}{m_{Z^{\prime}}^{4}} \quad\left(10 v_{Z^{\prime}}^{4}+22 v_{Z^{\prime}}^{2} a_{Z^{\prime}}^{2}+7 a_{Z^{\prime}}^{2}\right)
$$




$$
\begin{aligned}
\chi_{\infty}^{2}\left(e^{-} e^{-}\right) & =\mathcal{L} \quad \frac{\pi \alpha^{2} s}{m_{Z^{\prime}}^{4}} \quad\left(16 R_{Z^{\prime}}^{4}+16 L_{Z^{\prime}}^{4}\right) \\
& =\mathcal{L} \quad \frac{\pi \alpha^{2} s}{m_{Z^{\prime}}^{4}} \quad\left(32 v_{Z^{\prime}}^{4}+192 v_{Z^{\prime}}^{2} a_{Z^{\prime}}^{2}+32 a_{Z^{\prime}}^{2}\right)
\end{aligned}
$$

These Cramer-Rao minimum variance bounds are the best results one can hope to ever achieve in the absence of systematic errors for a given luminosity, center of mass energy and $Z^{\prime}$ mass. They may therefore serve as a benchmark of each reaction, and clearly indicate that the $e^{-} e^{-}$experiment can be more sensitive to small values of $v_{Z^{\prime}}$ and $a_{Z^{\prime}}$, especially in the diagonal (chiral) directions.

To be more realistic we impose from now on a cut of $10^{\circ}$ on the scattering angle and consider $90 \%$ polarized electron beams (i.e., a 5\% contamination of electrons with opposite helicity). Moreover, we assume the $e^{-} e^{-}$mode can only achieve half the luminosity of the $e^{+} e^{-}$mode. The relevant parameters at LEP2 and SLC $e^{-} e^{-}$as well as at $\mathrm{FLC}^{ \pm} e^{-}$are summarized in Tables 11 and 2.

Since the $e^{-} e^{-}$reaction provides very large statistics, it is important to reduce as much as possible the systematic errors. This can be done by normalizing the event numbers in each bin with respect to the total number of events. This way, we eliminate the by far dominant systematic error, which originates from the luminosity monitoring ${ }^{\square}$. The information from the total rates is then lost, but we already noticed from Fig. 2 that this observable is anyway not particularly relevant for the $Z^{\prime}$ signal in $e^{-} e^{-}$scattering.

The $e^{+} e^{-}$total cross section, on the other hand, is rather sensitive to the presence of a $Z^{\prime}$ and we should therefore keep this observable in the analysis. This goes without problem, because the event rates are sufficiently low to prevent the systematic error, which is mainly due to the luminosity measurement, to become dominant.

To set realistic observability limits on the $Z^{\prime}$ parameters we use the following two least squares estimators:

$$
\begin{aligned}
& \chi_{e^{+} e^{-}}^{2}=\sum_{\text {bins }}\left(\frac{n_{i}^{Z^{\prime}}-n_{i}^{S M}}{\Delta n_{i}^{S M}}\right)^{2} \quad\left(\Delta n_{i}\right)^{2}=n_{i}+\left(\epsilon_{\mathrm{syst}} n_{i}\right)^{2} \\
& \chi_{e^{-} e^{-}}^{2}=\sum_{\text {bins }}\left(\frac{n_{i}^{Z^{\prime}} / n^{Z^{\prime}}-n_{i}^{S M} / n^{S M}}{\Delta n_{i}^{S M} / n^{S M}}\right)^{2} \quad\left(\Delta n_{i} / n\right)^{2}=\frac{n_{i}}{n^{2}}\left(1-\frac{n_{i}}{n}\right),
\end{aligned}
$$

\footnotetext{
1 Note that in Møller scattering there is no systematic error due to electron-pion misidentification, as in Bhabha scattering.
} 
where we assume a systematic error $\epsilon_{\text {syst }}=1 \%$ on the $e^{+} e^{-}$luminosity measurement. Since the lepton angles can be measured to an accuracy better than $10 \mathrm{mrad}$, there is no systematic error due to bin-to-bin correlations, if we perfom the analysis with 10 bins. This way each bin is also guaranteed to contain a large number of events, which is almost gaussian distributed.

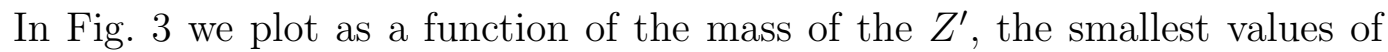
the reduced couplings $\left[{ }^{2} R_{Z^{\prime}} \sqrt{s} / m_{Z^{\prime}}\right.$ and $v_{Z^{\prime}} \sqrt{s} / m_{Z^{\prime}}$, which can be detected with $95 \%$ confidence $\left(\chi^{2}=6\right)$ at LEP2 or the SLC run in the $e^{-} e^{-}$mode. All collider parameters are summarized in Table 1. There is of course a $Z^{\prime}$ peak in the LEP2 $e^{+} e^{-}$reaction for $m_{Z^{\prime}}=\sqrt{s}=180 \mathrm{GeV}$. Obviously, close to this pole the $e^{+} e^{-}$mode provides the best information. For higher $Z^{\prime}$ masses and chiral couplings, the SLC $e^{-} e^{-}$can be more sensitive than LEP2.

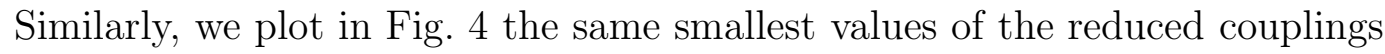
as a function of the mass of the $Z^{\prime}$, for the $e^{ \pm} e^{-}$modes of a $500 \mathrm{GeV}$ linear collider. The other collider parameters are summarized in Table 2. As anticipated, for $Z^{\prime}$ masses exceeding slightly the center of mass energy, the $e^{-} e^{-}$mode is better (even with only half the luminosity), especially for chiral couplings. As it turns out, these realistic predictions come within only a few percent of the of the theoretical limits (21,22) of each experiment.

\section{Resolving Power}

If a $Z^{\prime}$ is indeed discovered, it becomes interesting to measure as accurately as possible its couplings and mass. The same procedure as in the previous section can be applied for the parameter determination, replacing the standard model rates labeled $S M$ in Eqs (23,24), by the ones expected for the true values of the parameters, and computing the $\chi^{2}$ variations around these values. This is what we have done in Fig. 5, at a $500 \mathrm{GeV}$ collider for a $Z^{\prime}$ mass of $2 \mathrm{TeV}$ and for several possible values of the $Z^{\prime}$ vector and axial couplings.

For purely vector or axial $Z^{\prime}$ couplings, the $e^{-} e^{-}$reaction provides a better local resolution. For chiral couplings, though, both processes provide complementary constraints. This is mainly due to the fact that the $e^{-} e^{-}$mode cannot distinguish the four combinations of couplings related by the mirror symmetries around the diagonal axis $\left(R_{Z^{\prime}} \leftrightarrow-R_{Z^{\prime}}\right)$ or $\left(L_{Z^{\prime}} \leftrightarrow-L_{Z^{\prime}}\right)$. The $e^{+} e^{-}$reaction has only a two-fold ambiguity, relating combinations of couplings located opposite of the origin $\left(v_{Z^{\prime}}, a_{Z^{\prime}} \leftrightarrow-v_{Z^{\prime}},-a_{Z^{\prime}}\right)$. The use of $L R$ polarized beams does not improve the $e^{-} e^{-}$resolution beyond what can be

${ }^{2}$ We plot the couplings divided by the $Z^{\prime}$ mass only for rendering the asymptotic behaviour obvious. We also multiply by $\sqrt{s}$ to keep quantities dimensionless. 
achieved with $e^{+} e^{-}$.

To gauge the overall capabilities of linear collider, we have overlayed in Fig. 6 the expectations of the $Z^{\prime}$ models described in the second section with several $95 \%$ confidence contours. The latter have been obtained from the combined results of the $e^{+} e^{-}$and $e^{-} e^{-}$experiments, assuming a $Z^{\prime}$ mass of $1 \mathrm{TeV}$ and ignoring the ambiguity with respect to the central symmetry. For this $Z^{\prime}$ mass the picked models are thus all well distinguishable from each other and from the standard model.

As we can gather from Fig. 4 and Eqs (21,22), if the $Z^{\prime}$ is heavy compared to the collider energy, the couplings and the mass are correlated in such a way that it is difficult to disentangle a large mass from small couplings and vice versa. Asymptotically, for $m_{Z^{\prime}}^{2} \gg s$, the following scaling law applies

$$
\frac{\eta_{Z^{\prime}}}{m_{Z^{\prime}}}(s \mathcal{L})^{\frac{1}{4}}=\text { constant } \quad \eta_{Z^{\prime}}=v_{Z^{\prime}}, a_{Z^{\prime}}, R_{Z^{\prime}}, L_{Z^{\prime}}
$$

Having determined bounds on the couplings for a given energy, luminosity and $Z^{\prime}$ mass, it is trivial to use this scaling law to determine the corresponding bounds on the couplings for different energies, luminosities and $Z^{\prime}$ masses.

\section{Radiative Corrections}

We still have to address the issue of radiative corrections. They have been computed for the $e^{+} e^{-}$case [2] and turn out to degrade the $Z^{\prime}$ bounds by about $10 \%$. This is mainly due to initial state radiation which reduces the effective center of mass energy. Indeed, since the sensitivity of the $e^{+} e^{-}$reaction originates from probing the tail of the $Z^{\prime}$ peak, any reduction in energy lowers the signal to background ratio. This is clearly demonstrated by Figs 2, 1 .

In contrast, the $e^{-} e^{-}$process should not be much affected, because it probes the angular distribution, which is not significantly modified by radiative corrections in the considered angular range.

\section{Conclusions}

We have performed a comparison of $Z^{\prime}$ searches in the $e^{+} e^{-}$and $e^{-} e^{-}$modes of a linear collider of the next generation. For this, we used a similar analysis for each process and assumed the same collider parameters, except for the $e^{-} e^{-}$luminosity which we set to only half the $e^{+} e^{-}$luminosity. 
Both analysis procedures are rather efficient, yielding results very close to the theoretical Cramer-Rao limit. The $e^{-} e^{-}$mode turns out to be slightly better

for discovering a $Z^{\prime}$, while both reactions are complementary when it comes to determining the $Z^{\prime}$-lepton couplings.

For completeness, we have also compared the discovery potential of LEP2 vs SLC $e^{-} e^{-}$. Here also, both machines yield similar bounds, LEP2 being more performant for a light $Z^{\prime}$ and $\mathrm{SLC} e^{-} e^{-}$for a heavy $Z^{\prime}$.

\section{References}

[1] A comprehensive bibliography of high energy $e^{-} e^{-}$scattering can be found on the WWW at http://pss058.psi.ch/cuypers/e-e-.html.

[2] A. Leike, Z. Phys. C62 (1994) 265 hep-ph/9311356.

[3] D. Choudhury, F. Cuypers, A. Leike, Phys. Lett. B333 (1994) 531 hep$\mathrm{ph} / 9404362$.

[4] see for example

F. Del Aguila Acta Phys. Polon. B25 (1994) 1317 hep-ph/9404323;

S. Godfrey, Phys. Rev. D51 (1995) 1402 [hep-ph/9411237] and references therein.

[5] P. Langacker, M. Luo, Phys. Rev. D45 (1992) 278.

[6] H. Georgi, E. Jenkins, E.H. Simmons, Phys. Rev. Lett. 62 (1989) 2789; erratum 63 (1989) 1540; Nucl. Phys. B331 (1990) 541.

[7] R.N. Mohapatra, Unification and Supersymmetry, Springer, Berlin 1986.

[8] E. Ma, Phys. Rev. D36 (1987) 274;

K.S. Babu, X.G. He, E. Ma, Phys. Rev. D36 (1987) 878;

J.F. Gunion, A. Mendez, F. Olness, Int. J. Mod. Phys. A2 (1987) 118;

T.G. Rizzo, Phys. Lett. B206 (1988) 133.

[9] J.L. Hewett, T.G. Rizzo, Phys. Rep. 183 (1989) 193.

[10] F. Cuypers, Proceedings of the Workshop Perspectives for Electroweak Interactions in $e^{+} e^{-}$Collisions, Schloß Ringberg, 1995, World Scientific, Ed.: B. Kniehl hep-ph/9503252]. 
Table 1

\begin{tabular}{|c|c|c|}
\hline collider & LEP2 & $\mathrm{SLC} e^{-} e^{-}$ \\
\hline beams & $e^{+} \quad e^{-}$ & $e^{-} \quad e^{-}$ \\
\hline beam polarization [\%] & $0 \quad 0$ & $90 \quad 90$ \\
\hline energy $[\mathrm{GeV}]$ & 180 & 100 \\
\hline luminosity $\left[\mathrm{pb}^{-1}\right]$ & 10 & $2 \times 1$ \\
\hline polar angle cut $\left[^{\circ}\right]$ & 10 & 10 \\
\hline number of bins & 10 & 10 \\
\hline systematic error [\%] & 1 & 0 \\
\hline
\end{tabular}

Comparison of the different parameters used in the LEP2 and $\mathrm{SLC} e^{-} e^{-}$analysis.

Table 2

\begin{tabular}{|c|c|c|}
\hline collider & $\mathrm{FLC} e^{+} e^{-}$ & FLC $e^{-} e^{-}$ \\
\hline beams & $e^{+} \quad e^{-}$ & $e^{-}$ \\
\hline beam polarization [\%] & $0 \quad 90$ & $90 \quad 90$ \\
\hline energy [GeV] & 500 & 500 \\
\hline luminosity $\left[\mathrm{fb}^{-1}\right]$ & $2 \times 20$ & $2 \times 10$ \\
\hline polar angle cut $\left[{ }^{\circ}\right]$ & 10 & 10 \\
\hline number of bins & 10 & 10 \\
\hline systematic error $[\%]$ & 1 & 0 \\
\hline
\end{tabular}

Comparison of the different parameters used in the $\mathrm{FLC} e^{ \pm} e^{-}$analysis.
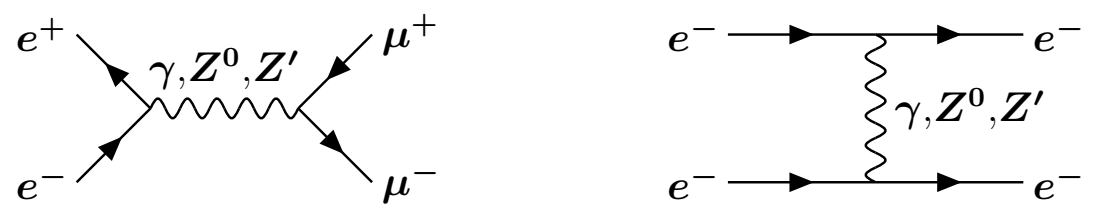

Fig. 1. Lowest order Feynman diagrams for the neutral gauge boson exchanges in $e^{+} e^{-}$and $e^{-} e^{-}$scattering. 


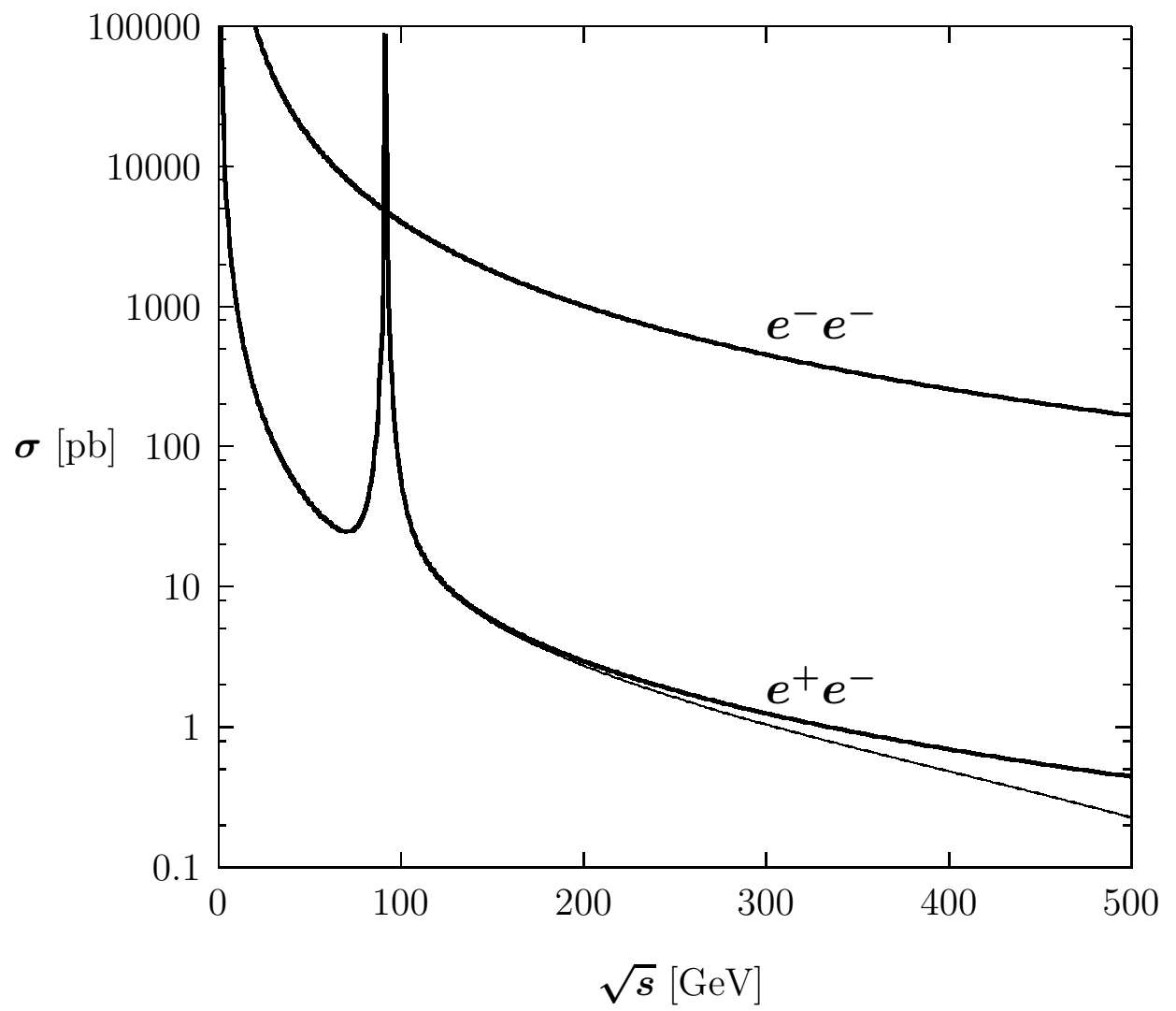

Fig. 2. Energy dependence of the unpolarized $e^{ \pm} e^{-}$cross sections in the framework of the standard model (thick curves) and with a $1 \mathrm{TeV} Z^{\prime}$ which couples like a photon (thin curves). In the $e^{-} e^{-}$case the two curves cannot be resolved on this scale. 


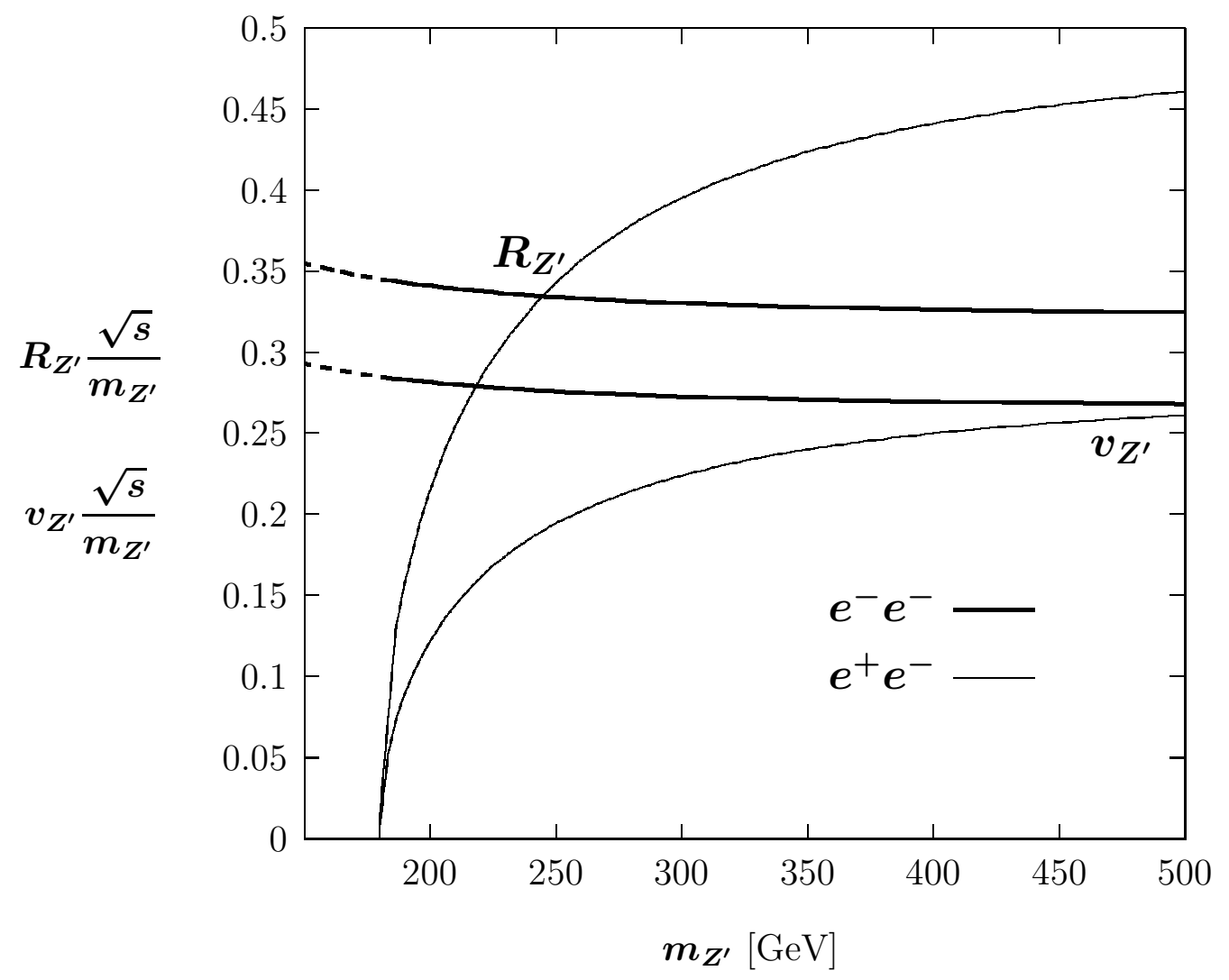

Fig. 3. Smallest measurable values of normalized $Z^{\prime}$ couplings with $95 \%$ confidence as functions of the $Z^{\prime}$ mass, at LEP2 and SLC $e^{-} e^{-}$(cf. Table 1). 


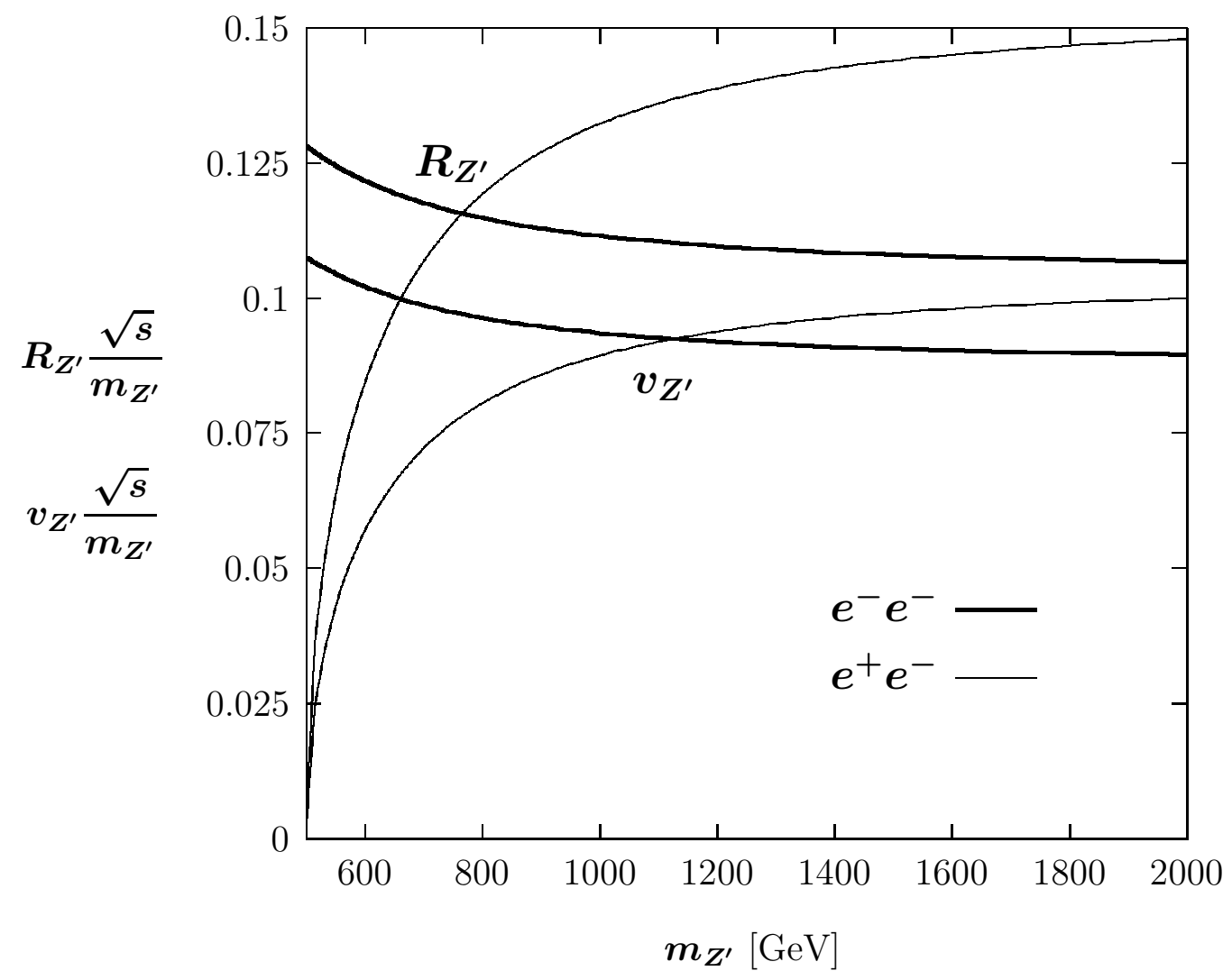

Fig. 4. Smallest measurable values of normalized $Z^{\prime}$ couplings with $95 \%$ confidence as functions of the $Z^{\prime}$ mass, in $e^{+} e^{-}$and $e^{-} e^{-}$collisions at the FLC (cf. Table 2). 


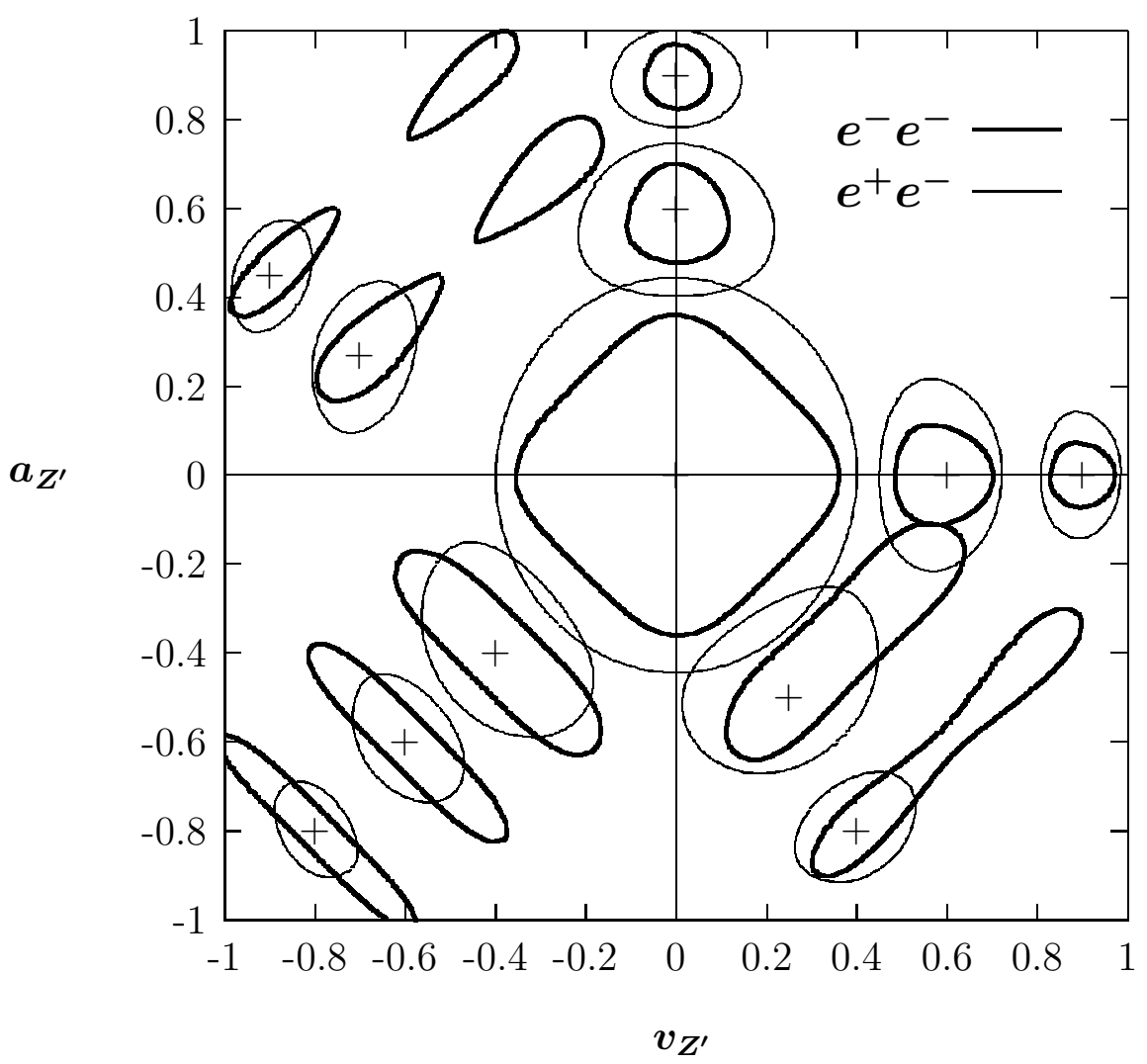

Fig. 5. Contours of resolvability at $95 \%$ confidence of the $Z^{\prime}$ couplings around several possible true values marked with a ' + '. The FLC characteristics are summarized in Table 2 and the $Z^{\prime}$ mass is $2 \mathrm{TeV}$. 


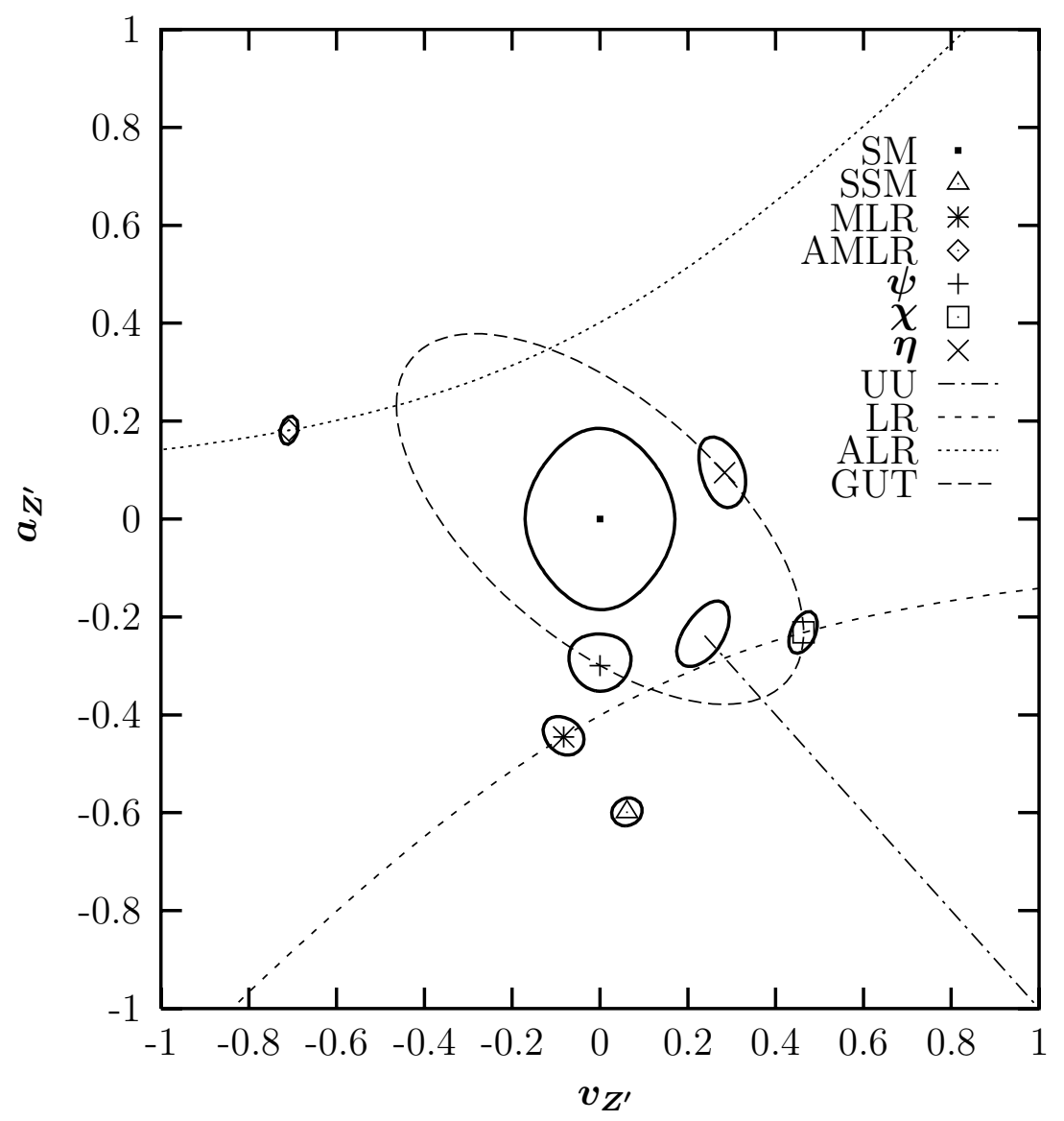

Fig. 6. Contours of resolvability at $95 \%$ confidence of the $Z^{\prime}$ couplings around several possible model predictions. The information from $e^{+} e^{-}$and $e^{-} e^{-}$collisions is combined. The FLC characteristics are summarized in Table 2 and the $Z^{\prime}$ mass is $1 \mathrm{TeV}$. 